\title{
La paradoja mexicana: de la evaluación de la calidad técnica de las elecciones de 2012, al debate sobre la calidad del voto y a la cuestión de la legitimidad democrática
}

\section{Willibald Sonnleitner* Arturo Alvarado** Arturo Sánchez***}

\section{Sumario:}

I. Introducción: La paradoja mexicana: elecciones con opciones, pero con un déficit creciente de legitimidad

II. Elecciones cada vez más competidas, plurales y precisas; pero cada vez más sospechosas, rechazadas e impugnadas

III. Principales hallazgos y conclusiones de la investigación

IV. Fuentes de consulta

* Profesor investigador en el El Colegio de México, donde enseña Sociología Política y Sociología Electoral. Desde 1998 ha observado y analizado dieciséis procesos electorales en América Latina.

** Profesor investigador y director del Centro de Estudios Sociológicos de El Colegio de México. Ha sido profesor visitante en las universidades de Harvard, El Instituto Tecnológico de Massachusetts y en la Universidad de La Sorbona en Francia.

*** Investigador asociado en El Colegio de México; sociólogo por la Universidad Autónoma Metropolitana y maestro en Estudios Latinoamericanos por la Universidad de Oxford. Fue designado como consejero electoral para el periodo 2003-2010.

D. R. () 2013. PNUD-UNAM, Instituto de Investigaciones Jurídicas. Revista Mexicana de Derecho Electoral, Especial sobre Observación Electoral 2012, núm. 4, pp. 369-392, México, D. F. 
Esta revista forma parte del acervo de la Biblioteca Jurídica Virtual del Instituto de Investigaciones Jurídicas de la UNAM www.juridicas.unam.mx

\section{Resumen:}

Este trabajo presenta los resultados del proyecto de observación académica de las elecciones del 1 de julio de 2012, realizado en el Centro de Estudios Sociológicos de El Colegio de México (CES-COLMEX), en el eje temático “Justicia Electoral” de la convocatoria del Programa de las Naciones Unidas para el Desarrollo (PNUD).

Parte del problema central de la pasada contienda presidencial se refiere, como se demuestra, de una elección competida y plural, con resultados técnicamente precisos y confiables; sin embargo, se trata de una elección cuyos resultados fueron impugnados de nueva cuenta en el marco de un polémico conflicto poselectoral, por lo que sufren de un importante déficit de legitimidad.

Esta situación paradójica se aborda desde tres perspectivas distintas en tres estudios complementarios: (1) la evolución de la calidad técnica de los resultados entre 1991 y 2012; (2) las razones del incremento inusual de las impugnaciones y las dinámicas de los conflictos poselectorales en los comicios federales y locales de 2012; y (3) los efectos multifacéticos de la violencia e inseguridad sobre los comicios y la calidad del voto.

Palabras clave: elecciones en México, conflictos poselectorales, calidad del voto, legitimidad, calidad de la democracia 


\section{Introducción. La paradoja mexicana: elecciones con opciones, pero con un déficit creciente de legitimidad}

La evaluación técnica de la calidad de las elecciones y la percepción social de su legitimidad resultan paradójicas en México. Hoy en día pocos países del mundo cuentan con sistemas tan sofisticados y confiables de organización electoral; a su vez, pocas sociedades son tan críticas, escépticas y desconfiadas al momento de reconocer los resultados de las contiendas democráticas.

Desde hace dos décadas la organización de los comicios institucionales asocia activamente a cientos de miles de ciudadanos independientes en la apertura y la atención de las casillas electorales, en la recepción y en el escrutinio de los sufragios, así como en la elaboración y en la entrega de las actas que dan constancia legal de sus resultados. Todas estas actividades son realizadas por personas seleccionadas al azar, debidamente capacitadas por el Instituto Federal Electoral (IFE) y ampliamente comprometidas con la democracia del país, en el marco de un proceso coordinado rigurosa y detalladamente por una institución autónoma, bajo el estrecho control de todos los partidos contendientes y bajo el escrutinio constante de la opinión pública y de los medios de comunicación.

Sin embargo, gran parte de la población mexicana duda actualmente de la calidad de sus procesos electorales, cuestionan abiertamente su elevado costo y manifiestan públicamente reservas sobre la confiabilidad de sus resultados. Como lo veremos enseguida, dicha percepción no solamente está difundida entre los sectores más desinformados y despolitizados de la ciudadanía, sino que también es compartida por algunos segmentos de las élites políticas, económicas y culturales del país, incluyendo académicos e intelectuales con una considerable influencia como líderes de opinión. De ahí el carácter polarizado y polémico de los debates que se siguen dando sobre la confiabilidad y la legitimidad de las elecciones en México. ¿Cómo estudiar esta paradoja desde una perspectiva empírica? 
Esta revista forma parte del acervo de la Biblioteca Jurídica Virtual del Instituto de Investigaciones Jurídicas de la UNAM

\section{Elecciones cada vez más competidas, plurales y precisas; pero cada vez más sospechosas, rechazadas e impugnadas}

A diferencia de los comicios de fachada que suelen organizarse en la mayoría de los regímenes autoritarios, las elecciones auténticas y democráticas, cuando éstas funcionan eficientemente, no solamente proporcionan opciones efectivas a los ciudadanos, sino que fungen como verdaderas fábricas de legitimidad: permiten seleccionar a los gobernantes entre una pluralidad de aspirantes con proyectos e intereses encontrados; pero permiten despejar, sobre todo, a los candidatos perdedores. En efecto, el reconocimiento explícito de los ganadores por los derrotados resulta crucial para legitimar a los gobernantes, dotándolos de una amplia aprobación popular y del beneplácito de todas las fuerzas políticas contendientes.

A la luz de esta premisa elemental, las pasadas elecciones en México se desarrollaron en una forma contra-productiva. A pesar de contar con uno de los sistemas de organización y escrutinio electoral más costosos, sofisticados y transparentes del mundo - que garantiza contiendas cada vez más competidas, plurales y precisas-, una parte sustantiva de la sociedad mexicana pone abiertamente en duda la calidad de los procesos electorales y desconfía de sus resultados. Como botón de muestra, según una encuesta publicada por el periódico Reforma el 12 de julio de 2012:

El 55 por ciento de los electores considera que las elecciones del 1 de julio fueron muy o algo limpias, aunque 40 por ciento cree que fueron muy o algo sucias. En cuanto a la organización de las mismas, el 51 por ciento la califica favorablemente, pero 48 por ciento le da notas de regular o mal... No obstante, el 55 por ciento dijo estar satisfecho con el resultado que arrojaron los comicios y 76 por ciento opina que López Obrador, quien ha tomado una postura de no reconocer dichos resultados, debería aceptarlos. $^{1}$

1 Moreno, Alejandro, "Encuesta Reforma: postelectoral. Deja dudas limpieza de los comicios, pero pide mayoría acatar resultados", Reforma, 12 julio de 2012 (énfasis añadido). 
Esta revista forma parte del acervo de la Biblioteca Jurídica Virtual del Instituto de Investigaciones Jurídicas de la UNAM

Esta desconfianza manifiesta hacia las elecciones no es nueva y tampoco resulta de consideraciones exclusivamente racionales, mucho menos de un conocimiento detallado de la forma como se organizan las elecciones por parte de las autoridades electorales. Tiene profundas raíces históricas, sobre las que volveremos a lo largo de esta investigación. También es alimentada por muchos medios de comunicación que, empezando con las mismas televisoras, se han dedicado a desacreditar al IFE desde la reforma electoral de 2007, responsabilizándolo de fallas que frecuentemente rebasan sus atribuciones legales. ${ }^{2}$

Pero los cuestionamientos más preocupantes no provienen de los medios, ni de la opinión pública, ni de la proporción de ciudadanos que dudan de la calidad de las elecciones. Provienen de las mismas élites y permean toda la clase política, lo que se traduce en un incremento inusitado de las impugnaciones y de los conflictos poselectorales, e incluso en el rechazo abierto de los resultados por algunos de los candidatos perdedores.

Eso sucedió, como es bien sabido, en las polémicas elecciones presidenciales de 2006, cuyo carácter extremadamente reñido (con una diferencia de solamente 0.56 puntos porcentuales entre los dos candidatos más votados), abrió una gran incertidumbre sobre la identidad del ganador, sobre el grado de precisión y sobre la confiabilidad misma de los resultados. Y lo mismo acaba de ocurrir de nuevo, durante las elecciones de julio de 2012 que, a pesar de saldarse por un margen de diferencia de 6.7 puntos porcentuales, fueron impugnadas nuevamente por la coalición Movimiento Progresista (MP).

Pero mientras que hace seis años, Andrés Manuel López Obrador (AMLO) y sus seguidores exigían, antes que nada, un recuento voto por voto, casilla por casilla, con el argumento de incrementar la certeza de los resultados de la elección, en esta ocasión el MP impugnó la constitucionalidad de los comicios, con base en la inequidad del proceso en su conjunto y las limitaciones a la misma libertad del voto de sendos millones de ciudadanos.

2 Como lo señala José Woldenberg, la mala prensa de la que sufre el organismo electoral desde 2007 no se relaciona necesariamente con las reglas del juego político-electoral. Se inscribe en el marco de una batalla mucho más amplia por el control del mercado de las telecomunicaciones y de los medios masivos de comunicación, en la que se encontró enfrascado el IFE por las nuevas atribuciones que recibió tras las últimas reformas electorales en materia de tiempos de difusión y compra de propaganda. Comunicación personal durante el Seminario sobre las elecciones de 2012, organizado por la Facultad de Ciencias Políticas de la UNAM, el 9 de mayo de 2012. 
Esta revista forma parte del acervo de la Biblioteca Jurídica Virtual del Instituto de Investigaciones Jurídicas de la UNAM

Ciertamente, este cambio notable de enfoque estratégico no implica que se reconozca la confiablidad de los resultados. Todo lo contrario: en 2006, 20377 casillas fueron atacadas formalmente ante los tribunales; ${ }^{3}$ esta vez, los juicios de inconformidad impugnaron 82493 casillas en la sola elección presidencial de $2012 .{ }^{4}$ A éstas se agregaron 141 impugnaciones en las legislativas federales, mediante un número igual de juicios de inconformidad. En el último caso, las sentencias del tribunal tuvieron consecuencias en los resultados del distrito 06 del Estado de México, donde se anularon 13 casillas y cambió el ganador. En cuanto a la elección presidencial, en ésta también se recontaron 1125 casillas mediante sentencia interlocutoria y procedió la anulación de 524 casillas, pero ello no tuvo efectos sobre el resultado. ${ }^{5}$

A estos juicios federales de inconformidad se suman las impugnaciones registradas en las elecciones locales para gobernador, diputados locales y ayuntamientos. Al respecto, nuestro seguimiento detectó al menos: 392 juicios de nulidad para la elección de diputados de mayoría relativa y representación proporcional en el Distrito Federal; 19 recursos de impugnación (uno por distrito electoral local) de la elección de gobernador, 48 en contra de los resultados de presidencias municipales y 4 de diputados de mayoría relativa y representación proporcional en Yucatán; 17 juicios de inconformidad en contra de la elección de gobernador, 13 en contra de los resultados de presidencias municipales y 3 en contra de los resultados de diputados de mayoría relativa en Tabasco; así como 120 juicios de inconformidad que engloban tanto las elecciones de ayuntamientos y diputados de mayoría relativa y representación proporcional, así como 64 recursos de apelación en el Estado de México. ${ }^{6}$

Más preocupante aún: lo que se impugna centralmente, en el nuevo enfoque estratégico, ya no se limita a irregularidades precisas acaecidas durante la jornada electoral, el escrutinio de los votos o el cómputo distrital; se extiende y se generaliza hacia la calidad y la inequidad

3 Se analizaron los 376 juicios de inconformidad en contra de los resultados de la elección de presidente de los Estados Unidos Mexicanos en 2006, para determinar el número de casillas que fueron impugnadas y anuladas.

${ }^{4}$ Boletín de Prensa del Tribunal Electoral del Poder Judicial de la Federación (TEPJF), publicado el 24 de agosto de 2012 http://portal.te.gob.mx/prensa/boletin-prensa/resuelvetepj-la-totalidad-los-juicios-inconformidad-el-computo-distrital-la-eleccio.

5 Idem.

${ }^{6}$ Información Global al pie de la página del Tribunal Electoral del Estado de México, www.teemmx.org. $m x$. 
del conjunto del proceso electoral. Al respecto, el contraste cualitativo entre las dos últimas impugnaciones presidenciales resulta revelador: mientras que en 2006, la Coalición por el Bien de Todos (CBT) solicitaba la reparación de irregularidades precisas relacionadas con el cómputo de los sufragios, en 2012 el juicio de inconformidad presentado por el MP exige directamente la anulación de toda la elección presidencial (como lo estipula el llamado Decálogo de Andrés Manuel López Obrador).

Sin embargo, pese a que dicho juicio de inconformidad se enfoque en una impugnación mucho más amplia de la inequidad de las elecciones para demostrar su invalidez constitucional, éste también cuestiona la certeza del escrutinio de los votos, asumiendo y sugiriendo que las incongruencias registradas en numerosas actas de casilla contribuyen a comprobar la irregularidad de los comicios presidenciales. De ahí la necesidad de analizar la calidad técnica de las pasadas elecciones.

\section{La calidad técnica de las elecciones bajo la lupa}

Para evaluar la calidad técnica de las elecciones federales de 2012, resulta interesante partir de las cinco impugnaciones sustanciales que cuestionan la confiabilidad y certeza de sus resultados. Éstas forman parte de un conjunto mucho más extenso de quejas y agravios que integran el juicio de inconformidad presentado por el Movimiento Progresista, por lo que merecen ser recordadas puntualmente.

¿Por qué se registran diferencias importantes entre las listas nominales del PREP y del cómputo distrital?, ¿cuál es la magnitud, y cuáles son los efectos políticos concretos de las votaciones atípicas que se registran en ambas bases de datos?, ¿qué efectos tuvo el recuento realizado por el IFE de más de 78000 casillas, sobre los resultados del cómputo distrital?, ¿cómo cuantificar y explicar las diferencias observadas entre los totales de votos en las elecciones para presidente, diputados y senadores?, y ¿hasta qué punto el incremento anormal y sospechoso de la lista nominal en las casillas rurales se conjuga con un comportamiento electoral atípico, tal y como se señala en el punto 10 del famoso Decálogo de AMLO?

Para abordar estos interrogantes, esta investigación parte de una cuidadosa exploración estadística de las distintas inconsistencias re- 
Esta revista forma parte del acervo de la Biblioteca Jurídica Virtual del Instituto de Investigaciones Jurídicas de la UNAM

gistradas en las 143130 casillas instaladas, relacionándolas con otros indicadores de la calidad y competitividad de los comicios, y controlada por los conocidos efectos de las variables sociodemográficas y contextuales. Una vez analizadas estas cinco impugnaciones, procedemos a una evaluación más amplia de la calidad técnica de los comicios de 2012, para contrastarlos con la calidad de las otras siete elecciones organizadas por el IFE desde 1991.

Para ello, partimos de una distinción básica - pero fundamentalentre las inconsistencias y las irregularidades que suelen producirse en todo proceso electoral. Mientras que las primeras se relacionan frecuentemente con simples errores humanos por lo que su distribución y sus efectos suelen ser aleatorios, las segundas remiten a manipulaciones deliberadas y proscritas en vista de alterar los resultados por lo que su distribución sí puede revelar acciones concertadas y dolosas de fraude.

De ahí el interés de estudiar el conjunto de inconsistencias que se produjeron durante las elecciones de julio de 2012 en las 143130 casillas electorales. ¿Cuán extendidas/excepcionales, y de qué naturaleza, fueron dichas incongruencias en estas últimas elecciones presidenciales?, ¿se limitaron éstas a ciertas regiones particulares, o caracterizaron el conjunto de entidades, distritos y municipios del país?, ¿se debieron todas las inconsistencias registradas a irregularidades y manipulaciones dolosas, o existen otros factores y elementos que permiten explicarlas?, ¿cuán importantes fueron los efectos político-electorales potenciales de las diversas inconsistencias, y de las posibles irregularidades?, y ¿qué revelan todos estos datos sobre la situación actual y sobre la evolución histórica de la calidad técnica de las elecciones en México?

En vistas de aportar elementos sólidos de respuesta, partimos de la identificación de inconsistencias graves (las casillas zapato, y aquellas en las que las tasas de participación rebasan el número total de ciudadanos inscritos en las listas nominales). Pero nos interesamos, también, en aquellos comportamientos electorales atípicos que pueden indicar eventuales irregularidades en el contexto particular de alguna sección electoral (tasas anormales de participación, cuasi unanimidad o hegemonía monopartidista, ausencia o exceso de votos anulados y/o determinantes, etcétera).

Acto seguido, relacionamos estas inconsistencias entre ellas, así como con otros indicadores complementarios que permiten controlar 
los efectos de otros factores político-institucionales (magnitud demográfica de las casillas, alianzas totales o parciales, etcétera) y sociodemográficos (niveles de urbanización, dimensiones constitutivas del índice de desarrollo humano [analfabetismo, escolaridad, salud y bienestar socioeconómico], y tasas de hablantes de lenguas indígenas).

Finalmente, elaboramos una tipología sociológica y una geografía comprensiva de las inconsistencias y de los comportamientos atípicos, en vistas de identificar las regiones en las que se ubican/concentran aquellas casillas y secciones que rompen con las tendencias normales. Ello permite comprender cómo se relacionan las distintas inconsistencias entre ellas, en qué medida obedecen a configuraciones y patrones geográficos específicos (o si se distribuyen en una forma aleatoria), e identificar los territorios en los que se concentran los distintos tipos de comportamientos atípicos. También proporciona elementos tangibles basados en datos duros, sistemáticos y confiables, que pueden contribuir a clarificar algunas de las percepciones confusas de la opinión pública y a reducir la desconfianza difusa de amplios sectores de la ciudadanía.

\section{De la incertidumbre de la contienda a la impugnación de los resultados}

En la segunda parte de este informe, se completa el estudio de las inconsistencias electorales con el análisis cuantitativo y cualitativo del periodo previo a los comicios, de la jornada electoral, del cómputo distrital, de las impugnaciones, así como de las estrategias discursivas, mediáticas y jurídicas de los principales partidos y coaliciones. En su conjunto, estos elementos configuran los conflictos poselectorales y generan el contexto sociopolítico en el que serán elaboradas las sentencias, antes de ser emitidas por los tribunales. Con ello, se busca contribuir a evaluar la magnitud efectiva, la percepción social y el tratamiento mediático de los agravios poselectorales, así como la capacidad del sistema vigente de justicia para procesarlos en México.

¿Cuál es el papel de los partidos y qué impacto tienen sus estrategias político-electorales, discursivas, mediáticas y jurídicas de impugnación sobre la percepción pública y sobre la legitimidad social de las elecciones?, ¿cómo las instituciones del sistema de justicia asimilan, procesan y resuelven estos conflictos poselectorales en el marco de la 
Esta revista forma parte del acervo de la Biblioteca Jurídica Virtual del Instituto de Investigaciones Jurídicas de la UNAM

legislación vigente en la actualidad?, ¿cuenta dicho sistema con medios eficientes para procesar los agravios y las inconformidades poselectorales, y cómo se perciben las resoluciones que emite el Tribunal Electoral del Poder Judicial de la Federación (TEPJF)?

Para indagar en estos interrogantes, partimos de la dinámica peculiar de la impugnación presidencial, ampliamente cubierta y comentada en los medios masivos de comunicación. Lejos de satisfacer a la parte litigante, el fallo del Tribunal Electoral del Poder Judicial de la Federación (TEPJF) fue rechazado y cuestionado públicamente, mientras que AMLO declaró que no reconocería un poder ilegítimo surgido de la compra del voto y de numerosas violaciones a la ley.

Pero, ¿hasta qué punto la dinámica de la impugnación de la presidencial resulta única, y hasta qué punto no refleja un padrón más difundido y generalizado de conflicto poselectoral?, ¿se debe ésta simplemente a un liderazgo excéntrico y a un partido particular, o se inscribe más bien en una lógica más amplia y sistémica relacionada con los incentivos y las constricciones que forjan las estrategias de impugnación poselectoral?

Como lo mostramos en esta investigación, el énfasis excesivo que han puesto los analistas y los medios masivos de comunicación en la elección presidencial ha contribuido a ocultar el incremento generalizado de las impugnaciones y los conflictos poselectorales, que se observa tanto en las legislativas federales como en los comicios concomitantes para gobernadores, ayuntamientos y legisladores locales que se organizaron el mismo día en dieciséis entidades de la República. Para romper con la tentación de personalizar o de extrapolar la lógica particular del conflicto poselectoral presidencial, lo contrastamos con las dinámicas concomitantes de los diversos conflictos poselectorales que se produjeron en las legislativas federales, así como en algunos de los comicios locales.

Asimismo, analizamos de cerca cómo se fueron configurando y construyendo los distintos escenarios de reconocimiento, cuestionamiento e impugnación de los distintos resultados electorales: desde el contexto preelectoral (dudas abstractas y quejas/denuncias anticipadas); pasando por la misma secuencia de la jornada electoral (cómo se conocen, asumen y comunican los resultados, con base en qué instrumentos, quién se declara primero ganador, a qué horas, y qué hacen los perdedores respectivos: reconocen su derrota, se declaran ganadores ellos también, mantienen el silencio, dicen que esperarán los resultados 
Esta revista forma parte del acervo de la Biblioteca Jurídica Virtual del Instituto de Investigaciones Jurídicas de la UNAM

oficiales, como lo hizo AMLO); hasta las estrategias que acompañan la preparación de las impugnaciones y la descripción y cuantificación misma de las impugnaciones/juicios de inconformidad; así como, en los casos que ya existan, las sentencias que confirman/anulan los resultados (en ese caso, cuántas casillas se anulan y cuáles son).

El análisis del proceso mediante el cual el Tribunal Electoral del Poder Judicial de la Federación emite su sentencia (los argumentos esgrimidos primero por el IFE, luego por la Coalición Movimiento Progresista y finalmente por el tribunal mismo) y la estrategia mediática seguida por los actores, permitió analizar las características del marco jurídico que debía aplicarse en 2012 y el marco interpretativo que aplicó el Tribunal para fundar y motivar su veredicto. Este aspecto resulta relevante, no sólo por constituir un precedente jurídico para próximos procesos, sino por las lecciones que pudieran surgir en el marco de una reforma electoral en ciernes.

\section{De la calidad de las elecciones a la calidad de los votos}

Para terminar, tanto la evaluación de la calidad técnica de los comicios como el incremento del número y el alcance de las impugnaciones poselectorales obligan a reflexionar, no sólo sobre la calidad de la contienda, de las campañas y del conjunto del proceso electoral, sino sobre la calidad misma del voto y de los contenidos del comportamiento político-electoral. Tras la histórica alternancia presidencial del año 2000, en México se dio frecuentemente por sentado que el sufragio se practica siempre en condiciones idóneas, de perfecta libertad e igualdad.

Como lo ilustran las numerosas manifestaciones e impugnaciones poselectorales, esa percepción optimista se pone ahora radicalmente en duda. ¿Cuán libre e igualitario fue el voto de los mexicanos en 2012, y hasta qué punto su posible compra y coacción limitaron su ejercicio efectivo?, ¿qué efectos tuvieron los diversos hechos de violencia sobre las campañas y los procesos electorales, y hasta qué punto incidieron en los resultados en un contexto de creciente inseguridad?

Sin poder realizar un estudio exhaustivo de esta dimensión compleja del voto por limitaciones de tiempo y recursos, nuestra investigación exploratoria presenta finalmente algunos elementos empíricos, acompañados de reflexiones e hipótesis sobre las relaciones entre la 
Esta revista forma parte del acervo de la Biblioteca Jurídica Virtual del Instituto de Investigaciones Jurídicas de la UNAM

violencia y la política electoral, para interrogarse sobre las condiciones heterogéneas en que se ejerce el voto en una sociedad marcada por la desigualdad y la inseguridad. Porque si bien resulta prematuro afirmar que la violencia ya ha desbordado los espacios institucionales que garantizan las condiciones elementales del sufragio universal, también resulta patente que en algunas regiones, municipios y contextos específicos, ésta sí está teniendo efectos tangibles sobre la calidad del proceso electoral.

\section{Principales hallazgos y conclusiones de la investigación}

Sinteticemos ahora los principales hallazgos, y las conclusiones más relevantes de esta investigación. Ambos permiten esclarecer la paradoja mexicana, e invitan a abrir un debate plural y crítico sobre los alcances, las limitaciones y los desafíos pendientes del proceso de democratización electoral en México. Éste debe partir del reconocimiento de las mejoras sustantivas realizadas en materia de organización, pluralidad y competitividad de las elecciones, pero también tiene que abordar cuestiones más amplias y complejas que atañen a las condiciones materiales en las que se ejerce el voto en la actualidad, lo que obliga a interrogarse sobre la cuestión crucial de su percepción y legitimidad social.

1. Elecciones cada vez más precisas, plurales, competitivas y confiables

Desde 1991, tanto la cantidad y el perfil sociodemográfico, como la concentración geográfica, el sesgo político-electoral y el nivel de asociación entre los comportamientos atípicos que se registran en el nivel de las casillas/secciones han pasado de magnitudes y características que ponían en duda la calidad técnica de las elecciones en vastas regiones del país, a magnitudes y a características que sitúan los comicios federales de 2012 en un nivel radicalmente distinto, de pluralidad y competición generalizadas, y de elevada precisión y confiabilidad. 
Esta revista forma parte del acervo de la Biblioteca Jurídica Virtual del Instituto de Investigaciones Jurídicas de la UNAM

Ciertamente, persisten casillas con comportamientos anormales y sospechosos, e incluso algunos casos con patentes irregularidades: entre ellos, nueve casillas zapato y 88 casillas con tasas de participación superiores al $100 \%$ de los ciudadanos inscritos. Asimismo, algunas inconsistencias guardan claros sesgos político-partidistas y siguen beneficiando a los partidos que tienen mayor arraigo en las zonas más apartadas, donde escapan a la competencia plural y al control efectivo de representantes de partidos debidamente capacitados y motivados.

Para dar un botón de muestra, las prácticas corporativas de control, acarreo y coacción del voto persisten al menos en las 2712 casillas (1.9\% del total) que todavía se encuentran bajo la férula de alguna fuerza hegemónica (es decir, con un solo partido político efectivo), y/o incluso dominante (en 282 casos, el partido más importante capta más del $90 \%$ de los votos válidos). En su mayoría, estas situaciones monopartidistas benefician a Enrique Peña Nieto (EPN), aunque en menor medida también a Andrés Manuel López Obrador, mientras que afectan negativamente a Josefina Vásquez Mota (JVM) y a Gabriel Quadri (GQ).

Sin embargo, en 2012 ningún partido tiene el monopolio de dichas prácticas, como también lo refleja la existencia de casillas zapato y/o unánimes que votan en una forma disciplinada y cargada, más frecuentemente por candidatos del Partido Revolucionario Institucional (PRI), pero también por candidatos del Partido de la Revolución Democrática (PRD), del Partido Acción Nacional (PAN), del Partido del Trabajo (PT) y del Movimiento Ciudadano (MC). ${ }^{7}$ Ello depende, en buena medida, del tipo de implantación y de la estrategia de movilización que privilegian los distintos partidos en las distintas entidades y regiones del país, como lo ilustra la expansión reciente del PAN en algunas zonas rurales en las que nunca contó con militantes ni simpatizantes históricos, así como la existencia de sólidos bastiones rurales

\footnotetext{
7 Para dar otro ejemplo preciso: cuatro de las nueve casillas zapato contienen de hecho un solo voto, y una más contiene cuatro votos en total; de las cuatro restantes, tres están cargadas a favor de Enrique Peña Nieto (con 47, 74 y 76 votos unánimes, respectivamente), y la otra a favor de Andrés Manuel López Obrador (pero ésta con 206 votos unánimes). En suma, la anulación del conjunto de estas nueve casillas zapato afectaría muy ligeramente a AMLO y a EPN (haciéndoles perder 208 y 203 votos respectivamente), haciendo pasar la ventaja de EPN sobre AMLO de 6.78749\% a $6.78756 \%(+0.00007$ puntos porcentuales).
} 
Esta revista forma parte del acervo de la Biblioteca Jurídica Virtual del Instituto de Investigaciones Jurídicas de la UNAM

que el PRD u otros partidos lograron arrebatar, en un pasado más o menos reciente, al partido tricolor.

En cuanto a la ventaja más amplia del PRI en las casillas rurales, ésta se debe en mayor medida a la debilidad y/o a la fragmentación de los otros partidos competidores en las zonas más marginadas del país, que a un arraigo propio comparable con el que éste contó durante el periodo autoritario. Ello se refleja en el declive histórico y en la debilidad relativa del tricolor en el nivel de las 143413 votos. Al respecto, no hay que olvidar que, en 2012, EPN apenas captó 31.9\% de los votos válidos a través de su propio partido, sumando más de 6 puntos porcentuales adicionales a través de sus aliados del PVEM. Cuando se suman los votos presidenciales del PRI con la mitad de los votos que le corresponden por concepto de su alianza con el PVEM, se llega a la siguiente distribución de frecuencias en el nivel de casillas.

\section{Cuadro 1}

\begin{tabular}{|c|c|c|c|c|c|c|c|c|c|c|c|c|c|c|}
\hline 96PRI_1991-D & & & & & & \multicolumn{7}{|c|}{ percentiles } \\
\hline Válido5 & Perdidos & Media & Desv. típ. & Mínimo & Máximo & 1 & 5 & 25 & 50 & 75 & 90 & 95 & 99 \\
\hline 87333 & 289 & 63.7 & 18.6 & 0.0 & 100.0 & 23.5 & 36.8 & 49.3 & 62.5 & 77.7 & 90.4 & 95.8 & 100.0 \\
\hline \%PRI_2012-P & & & & & & \multicolumn{7}{|c|}{ percentiles } \\
\hline Válido5 & Perdidos & Media & Desv. típ. & Mínimo & Máximo & 1 & 5 & 25 & 50 & 75 & 90 & 95 & 99 \\
\hline 143413 & 926 & $\mathbf{3 4 . 2}$ & 11.4 & 0.0 & 100.0 & 12.6 & 18.0 & 26.1 & 33.4 & 40.7 & 48.6 & 54.4 & 68.1 \\
\hline
\end{tabular}

En un contraste radical con la situación que imperaba en 1991 cuando el tricolor controlaba al menos $62.5 \%$ del voto en la mitad de las 87000 casillas - en 2012 el PRI apenas obtuvo una tercera parte de los sufragios válidos en la mitad de las 143000 casillas. De hecho, sólo rebasó el 50\% de los votos en una de cada doce casillas, mientras que ello todavía era el caso en tres de cada cuatro casillas en 1991. Pero sobre todo sus resultados hegemónicos se concentran, ahora, en menos de $2 \%$ del total de casillas, mientras que 21 años atrás ese era el caso en $30 \%$ de las casillas.

En otras palabras, el margen holgado de 21.3 puntos porcentuales con el que EPN aventajó a AMLO en las casillas rurales ya no se relaciona con una situación de partido hegemónico o dominante. Se debe a la combinación de la mayor resistencia del tricolor en el campo (que le permitió captar a EPN 47.2\% del sufragio rural contra 25.9\% a favor de López Obrador), con una concentración inversa del voto de AMLO en las casillas urbanas (donde este último se benefició de un impor- 
Esta revista forma parte del acervo de la Biblioteca Jurídica Virtual del Instituto de Investigaciones Jurídicas de la UNAM

tante efecto de arrastre y sumó 34\% del sufragio, contra $37.2 \%$ a favor de Peña Nieto). Este efecto de arrastre tiene un perfil urbano y es particularmente palpable en las presidenciales, lo que indica que el voto cruzado se debe fundamentalmente a méritos propios de notabilidad personal de AMLO, que no generó la candidatura de EPN. En todo caso, no hay nada realmente atípico en ese tipo de comportamiento, al menos si uno se refiere al periodo histórico que se caracteriza por elecciones competitivas y plurales.

En suma, en 2012 el número de casillas con comportamientos atípicos es tan reducido que, incluso cuando resulta políticamente sesgado, apenas afecta el resultado electoral en su agregación nacional. En la situación que todavía imperaba en 1991, la hipotética anulación del conjunto de casillas con comportamientos monopartidistas hubiera modificado sustancialmente los resultados electorales en aquellas elecciones legislativas intermedias, al reducir el margen de victoria de $45.1 \%$ a $30.8 \%$ de los votos válidos (-14.3 puntos porcentuales). Este año, la anulación de las 2712 casillas que se mantuvieron bajo la hegemonía atípica de una sola fuerza política hubiera modificado, a su vez, el margen de victoria en 162579 votos, reduciéndolo de $6.79 \%$ a $6.54 \%$ (-0.24 puntos porcentuales).

\section{Cuadro 2}

\begin{tabular}{|c|c|c|c|c|c|c|c|c|c|}
\hline Elección 2012 & & EPN_Pr & AMLO_Pr & EPN-AMLO_Pr & JVM_Pr & GQT_Pr & Val_Pr & Nulos_Pr & Totales_Pr \\
\hline \multirow[t]{3}{*}{ Pluripartidistas } & $\mathrm{N}$ & 140,686 & 140,686 & 140,686 & 140,686 & 140,686 & 140,686 & 140,686 & 140,686 \\
\hline & Suma & $18,850,087$ & $15,682,881$ & $3,167,206$ & $12,720,385$ & $1,145,110$ & $48,398,463$ & $1,218,120$ & $49,637,295$ \\
\hline & $\%$ & 38.95 & 32.40 & 6.54 & 26.28 & 2.37 & 100 & & \\
\hline \multirow[t]{3}{*}{ Casillas Monopartidistas } & $\mathrm{N}$ & 2,712 & 2,712 & 2,712 & 2,712 & 2,712 & 2,712 & 2,712 & 2,712 \\
\hline & Suma & 376,697 & 214,118 & 162,579 & 66,262 & 5,552 & 662,629 & 21,981 & 684,805 \\
\hline & $\%$ & 56.85 & 32.31 & 24.54 & 10.00 & 0.84 & 100 & & \\
\hline \multirow[t]{4}{*}{ Total } & $\mathrm{N}$ & 143,398 & 143,398 & 143,398 & 143,398 & 143,398 & 143,398 & 143,398 & 143,398 \\
\hline & Suma & $19,226,784$ & $15,896,999$ & $3,329,785$ & $12,786,647$ & $1,150,662$ & $49,061,092$ & $1,240,101$ & $50,322,100$ \\
\hline & $\%$ & 39.19 & 32.40 & 6.79 & 26.06 & 2.35 & 100 & & \\
\hline & & EPN_Pr & AMLO_Pr & EPN-AMLO_Pr & JVM_Pr & GQT_Pr & & & \\
\hline Efectos relativos de la anulación & $\%$ & -0.242 & 0.001 & -0.243 & 0.220 & 0.021 & & & \\
\hline
\end{tabular}

En efecto, de los 684805 votos que hubieran sido anulados, 376697 hubieran sido deducidos de los 19.2 millones de Enrique Peña Nieto, mientras que una parte sustancialmente menor (66262 sufragios) hubieran sido deducidos de los 12.8 millones de votos captados por Josefina Vázquez Mota. Sin embargo, en la medida en la que AMLO registró porcentajes muy similares en estas casillas monopartidistas (32.3\%) que en el resto del país (32.4\%), la anulación también le hubiera costado 214118 sufragios. En otras palabras, las pérdidas de 
Esta revista forma parte del acervo de la Biblioteca Jurídica Virtual del Instituto de Investigaciones Jurídicas de la UNAM

EPN (-0.24 puntos) se hubieran traducido en ganancias marginales en beneficio de JVM (+0.22 puntos) y de GQ (+0.02 puntos), pero prácticamente no hubieran afectado el porcentaje de AMLO $(+0.001$ puntos).

Al tratarse exactamente del mismo tipo de comportamiento atípico analizado en 1991, podemos establecer una comparación aproximativa entre ambas elecciones. En 2012, el efecto político-electoral potencial de las casillas monopartidistas sobre el margen de victoria fue 59 veces menos importante que 21 años atrás, al situarse alrededor de 0.24 puntos porcentuales (contra 14.3 puntos en 1991). Ello proporciona una buena idea de los avances sustantivos realizados en materia de pluralismo, competitividad, precisión y confiabilidad técnica de las elecciones mexicanas.

Este ejercicio hipotético invita, así, a matizar el efecto potencial de este tipo peculiar de posibles irregularidades, al menos cuando las elecciones se deciden por márgenes de victoria amplios (como fue el caso en las presidenciales de 2012). Sin embargo, también ilustra la importancia determinante que éstos todavía pueden llegar a tener en contiendas de elevada competitividad, en las que eventuales irregularidades sí pueden adquirir efectos determinantes. Y este tipo de situaciones es mucho más frecuente de lo que suele pensarse. No solamente se produjo, como fue menester, en las elecciones presidenciales de 2006. Hubiera podido producirse en los comicios presidenciales de 2000, si el efecto de arrastre generado por la candidatura de Vicente Fox no hubiera ampliado la diferencia cerrada que separó la Alianza por el Cambio del PRI en las legislativas concomitantes (ésta fue entonces de solamente 1.4 puntos porcentuales).

Sin ir tan lejos, la creciente fragmentación del sistema partidista tiene como consecuencia que este tipo de contiendas se haya vuelto cada vez más recurrente en México, como lo ilustra un número importante de elecciones recientes de nivel de gobernador, diputados y presidentes municipales. Tal fue el caso de las elecciones para gobernador de Chiapas en 2006 (cuando la diferencia registrada por el PREP se situaba en 0.22 puntos porcentuales), así como en las elecciones locales de Michoacán en 2011 (que fueron anuladas en Morelia, la capital del estado de Michoacán de Ocampo).

Considerando las tendencias estructurales del sistema de partidos mexicano, todo indica que este tipo de situaciones continuará incrementándose en los años por venir. En esta perspectiva, resulta funda- 
Esta revista forma parte del acervo de la Biblioteca Jurídica Virtual del Instituto de Investigaciones Jurídicas de la UNAM

mental seguir investigando con mayor profundidad y detenimiento las dimensiones oscuras y ocultas de las elecciones, cuyas inconsistencias bien pudieran transformarse en fuentes de conflicto e incertidumbre en otras contiendas reñidas con resultados estrechos. De hecho, durante los mismos comicios federales y locales que se celebraron en julio de 2012, esta situación se produjo en varios estados, distritos y municipios, desembocando en un número inusitado de conflictos poselectorales. De ahí el interés de analizar la dinámica que siguieron los distintos procesos de impugnación de las elecciones.

\section{Entre la lucha por la defensa del voto y la construcción de la desconfianza para cuestionar la validez de las elecciones}

Para empezar, se registró un incremento significativo en el número de recursos que se presentaron ante el TEPJF a lo largo de todo el proceso electoral 2011-2013. En particular, destaca el aumento del número de recursos de apelación que pasó de 97 en el año 2006, a 659 en el año 2012. Si bien este tipo de recurso apela fundamentalmente contra las decisiones del Consejo General del IFE, y no contra los acontecimientos de la jornada electoral, los números muestran una tendencia creciente de recurrir a las instancias jurisdiccionales para protestar contra las decisiones de la autoridad. En general, la investigación muestra que existió un esquema de impugnación no sólo de los acontecimientos del 1 de julio, sino desde que inició el proceso electoral en octubre de 2011.

En el caso de las impugnaciones contra los resultados electorales, los denominados juicios de inconformidad, el incremento en relación con 2006 es también significativo. Dichos juicios pasaron de 495 a 520, aunque con una particularidad: en la medida en que dichos juicios se refieren a los cómputos distritales, difícilmente su número podría ser muy superior a los 300 (dado que ese es el número de distritos que existen). Sin embargo, es ahí en donde se concentraron los principales cuestionamientos a la elección.

El trabajo también muestra la importancia de la presencia de representantes de los partidos políticos en las casillas durante la jornada electoral y la calidad de su participación. El siguiente cuadro ilustra 
Esta revista forma parte del acervo de la Biblioteca Jurídica Virtual del Instituto de Investigaciones Jurídicas de la UNAM

que, si bien todos los partidos registraron un alto número de representantes, el porcentaje de ellos que permaneció durante todo el día y firmó el acta correspondiente al finalizar la jornada electoral fue sustantivamente menor.

\section{Cuadro 3}

\section{Registro y Firmas de los Representantes de los Partidos Políticos 2012.}

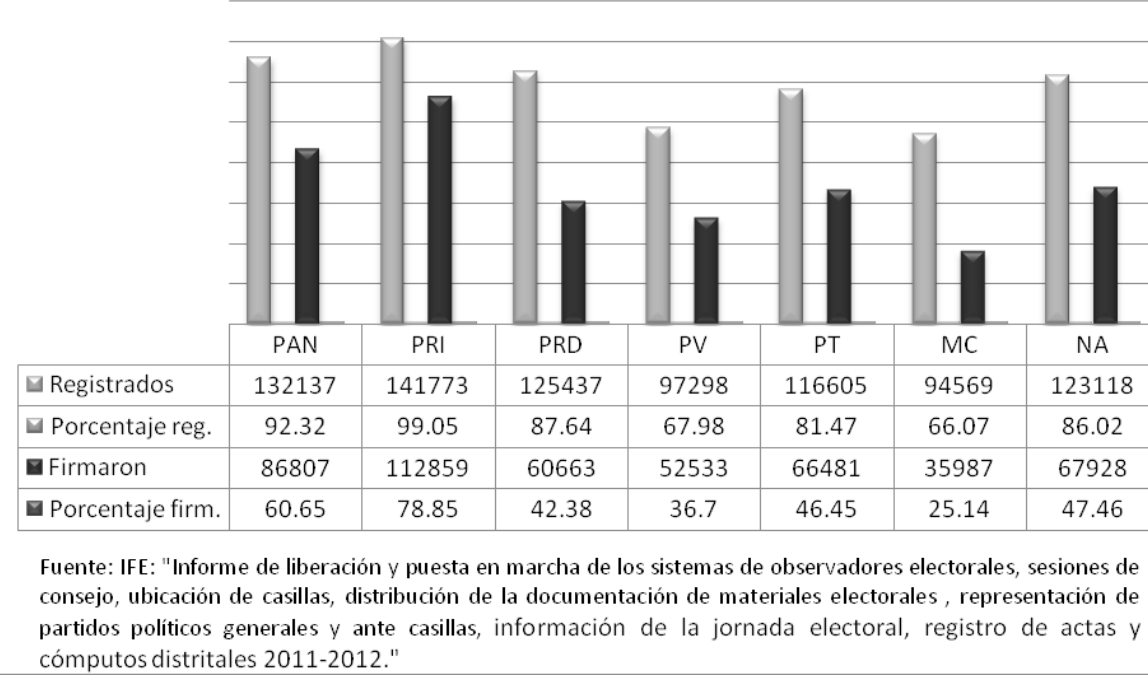

Con todo, la presencia de representantes de partidos constituyó una importante garantía para vigilar el proceso de la organización de la elección. Esa es una de las razones por las cuales en 2012, el peso de la impugnación no se centró en los eventuales problemas que se presentaron en las casillas. Sin embargo, la Coalición MP no dejó de cuestionar algunas de las características de la jornada electoral, en particular por las discrepancias en algunos datos, que fueron mencionados anteriormente.

El análisis de la impugnación denominada madre interpuesta por la Coalición MP, y que correspondió al juicio JIN-359, muestra una importante diferencia en relación con 2006. En aquel año, el objetivo de la impugnación consistía en tratar de revertir el resultado de la elección. De ahí la campaña de comunicación centrada en el voto por voto, casilla por casilla. En el proceso electoral de 2012, el objetivo no 
consistió en revisar el número de votos, sino en cuestionar la validez del conjunto de la elección con base en la falta de respeto a los principios constitucionales que garantizan la celebración de elecciones libres, auténticas y democráticas.

Por eso la dinámica de la impugnación requería de remontar los siguientes argumentos: 1) el tribunal tenía atribuciones suficientes para entrar al fondo de los temas, a pesar de que la ley establece que la nulidad es factible con base en causales explícitas en la ley, lo cual no era el caso; 2) la equidad en la contienda se había violado por la interferencia de los medios de comunicación en distintas partes del proceso electoral, incluso en la construcción de la candidatura del candidato ganador; 3) la inequidad también se había manifestado en la adquisición y en el uso de recursos económicos, mediante mecanismos que presuntamente violaron la ley, y 4) la libertad del sufragio se había violentado mediante la compra y la coacción de los ciudadanos a votar por el candidato del PRI y del PVEM.

A este tipo de impugnación correspondió un nuevo esquema de respuesta por parte del Tribunal Electoral. Resulta importante que el TEPJF dedicara las primeras 100 páginas a reiterar su carácter de tribunal constitucional y en consecuencia estuviera en facultades para entrar al fondo de los temas. Con esa base, desestimó todos los agravios pero sentó precedentes importantes en materia del valor de las pruebas que se presentan; el alcance de la libertad de expresión en las campañas y sobre el uso de los recursos por parte de los partidos.

Finalmente, resultó interesante observar que la dinámica de las impugnaciones en la elección federal no es propia ni de un partido político, ni de un candidato. Cuando se analizan las impugnaciones en los distintos niveles de elección (gobernador, legisladores federales y locales, ayuntamientos) y en las distintas entidades de la República que celebraron comicios locales, es factible observar cómo se reproducen los mismos esquemas y argumentos de quienes llegaron en segundo lugar en las preferencias electorales, independientemente de la distancia entre el primer y segundo lugar, de la orientación ideológica y/o de la adscripción partidista de los candidatos derrotados. 
Esta revista forma parte del acervo de la Biblioteca Jurídica Virtual del Instituto de Investigaciones Jurídicas de la UNAM

\section{Violencia, desigualdad e inseguridad versus democracia, libertad y equidad}

Finalmente, las elecciones de 2012 se desarrollaron en un país convulsionado por la inseguridad y ante los temores de que ésta contaminara el proceso electoral. Afortunadamente, ninguna situación durante la jornada electoral pudo caracterizarse como un episodio de violencia político-electoral. Las autoridades lograron organizar el proceso de una forma ordenada, y ofrecieron resultados en tiempo y forma. Los candidatos electos también lograron ocupar sus cargos, y el cambio de gobierno ha tenido lugar sin contratiempos.

No obstante, en el marco temporal más amplio del conjunto del proceso electoral, sí se observaron algunos eventos que, aunque no fueron sancionados, causaron incertidumbre y desconfianza por parte de los votantes. Si a esto le sumamos el elevado nivel de violencia que, sin irrumpir directamente en la jornada electoral, también pudo haber incidido previa o posteriormente a ella, obtenemos una mezcla de situaciones en la que los actores políticos (partidos y candidatos), los medios de comunicación y otros grupos de intereses pueden haber influido en las elecciones de maneras no siempre legítimas. Un ejemplo fue la renuencia de ciertos actores a desistir de realizar actividades a todas luces ilegales en su afán por conquistar el poder político, movilizando recursos para su enriquecimiento personal o para otras actividades que se alejan de los propósitos democráticos.

En efecto, la crónica impunidad y la falta de castigo crean incentivos para infringir la ley, sin importar las repercusiones jurídicas y penales, permitiendo en algunas ocasiones la incidencia directa de la delincuencia organizada y el narcotráfico sobre el proceso político local. Mientras que las elecciones federales se realizaron sin mayores dificultades, algunos de los comicios que tuvieron lugar en las entidades federativas durante el mismo periodo sufrieron diversos hechos de violencia político-electoral, que restringieron las libertades en algunas partes del territorio. Ya sea a través de la compra o la coacción del voto, la extorsión, la amenaza o hasta el asesinato de varios candidatos y ediles en funciones, varias elecciones locales se desarrollaron en condiciones muy adversas, y ello afectó la calidad de la democracia en nuestro país. 
Los órganos competentes procedieron a revisar el proceso electoral federal, y hasta la fecha no encontraron irregularidades mayores. Pero queda pendiente la denuncia de la compra de votos por medio de esquemas de tarjetas para comprar bienes (Monex y otras), que solamente será resuelta en enero de 2013, cuando las autoridades electas ya estén en funciones y sólo quede la opción de multarlas por eventuales actos que hubiesen alterado los resultados, minando por ende la legitimidad del proceso electoral.

El comportamiento de los partidos y de sus líderes frente a este tipo de casos refleja un escaso compromiso democrático. A través de sus representantes y legisladores, éstos son los creadores del andamiaje jurídico e institucional que regula los procesos electorales y, al mismo tiempo, son los que pueden violar las leyes electorales impunemente. En México, existe la costumbre de evadir la ley, ya sea vía el camuflaje de las acciones ilícitas o bien, a través de argumentos legaloides con los que se busca escapar de las sanciones legales.

Pero las actividades ilegales no son realizadas exclusivamente por bandas criminales, ni por partidos políticos. Otro tema importante en este proceso electoral fue la actuación de los medios de comunicación que, como lo creen amplios sectores descontentos, impusieron sus intereses y distorsionaron la calidad de la información durante las campañas electorales. Varios estudios ofrecen evidencias parciales al respecto, por lo que el papel de los medios en la construcción de una democracia más transparente e igualitaria quedará pendiente para futuras investigaciones.

Otro tema igualmente complejo es la denuncia sobre el uso y abuso de las encuestas. Este año, éstas fueron criticadas duramente por supuestas manipulaciones que, se aduce, limitaron la competitividad del proceso electoral. Ciertamente, la regulación del IFE ha mejorado notoriamente, pero sigue siendo insuficiente para generar legitimidad. El hecho que no se encontraran irregularidades en ninguna de las denuncias no significa que no existieran bases para realizar una investigación más profunda. Es entendible que el IFE cuente con capacidades institucionales limitadas (no obstante que la última reforma electoral le confirió más competencias) para resolver todas las supuestas irregularidades. Pero también se puede pensar que es posible revisar con más cuidado los agravios y otras posibles irregularidades denunciadas por la prensa y la ciudadanía. 
Esta revista forma parte del acervo de la Biblioteca Jurídica Virtual del Instituto de Investigaciones Jurídicas de la UNAM

Un caso incluso aún más complicado por estudiar es el clientelismo político, concepto en el que es difícil hallar un consenso sobre su significado, pero una práctica persistente que permea la política de nuestro país. En México, los dispositivos de intercambio clientelar escapan generalmente de la regulación jurídica, a menos de que trasciendan hacia otras actividades como el tráfico de influencias, el desvío de recursos públicos, la compra de votos, la corrupción, o el abuso de poder. Sin embargo, por estar fundamentados en relaciones asimétricas que instrumentalizan las necesidades de muchas personas (sin importar su posición económica), éstos atentan contra la igualdad y la libertad de los ciudadanos para elegir a sus representantes.

El clientelismo puede analizarse como una interfase entre actividades electorales y otras coercitivas, no democráticas e ilegales. Así podemos clasificar actos de favoritismo, compra de votos, reparto de bienes y servicios que, de hecho, es obligación de los gobiernos otorgar, promesas a cambio de favores, o condicionamientos de los servicios públicos para favorecer a un candidato o partido. Pero aunque la compra de votos está tipificada como delito electoral, muchas de sus modalidades escapan de las sanciones previstas en la ley, ya sea porque no es fácil comprobarlas o bien porque se disfrazan con la implementación de programas sociales.

Según un estudio de Alianza Cívica, hasta el 28.4\% de los ciudadanos estuvieron expuestos a alguna forma de compra o coacción del voto en las elecciones de 2012. De acuerdo con la asociación civil, la alianza Compromiso por México (PRI-PVEM) fue el actor mayormente beneficiado por estas prácticas, abarcando el $71 \%$ de los casos reportados por la organización civil. A su vez, para la Misión de Expertos Electorales (MEE) de la Unión Europea la actuación del IFE fue tibia ante las denuncias de compra y coacción de voto, calificándola incluso como "abiertamente conservadora y como una oportunidad perdida para emitir una señal de desaprobación hacia este tipo de prácticas". ${ }^{8}$

La pregunta que surge es por qué persisten, y cómo se reproducen las prácticas clientelares a lo largo del tiempo. A nivel local, estas son perpetuadas por gobernadores y presidentes municipales, quie-

8 Tal como puede apreciarse en el Informe Final, Elecciones generales 1 de julio de 2012, en http://www.ife.org.mx/docs/IFE-v2/CNCS/CNCS-IFE-Responde/2012/Octubre/I nfMEuro/InfMEuro.pdf consultada en diciembre 2012. 
nes operan electoralmente a favor de sus partidos a través de la implementación de programas públicos, como una forma de compra de voto cuasi institucionalizada. En ocasiones, los jueces han encontrado conductas ilícitas que alteran los procesos electorales, pero las consideran mínimas o no encuentran culpables; incluso, cuando los hay, se carece de medios para castigarlos. Así, el hecho que los partidos políticos o los candidatos rompan las reglas del juego, no produce sanciones sustantivas, como la persecución legal o la pérdida del registro, ni la anulación de la elección. En la mayoría de los casos éstos simplemente son multados, lo cual es pagado con recursos públicos. En otras palabras, son los contribuyentes los que terminan pagando por las irregularidades, ya que la élite político-partidaria mexicana no parece estar dispuesta a implantar un Estado de derecho que la constriña efectivamente.

Para terminar, la preocupación principal en materia de construcción democrática continúa siendo la inequidad, que limita la participación libre, igualitaria y abierta de la ciudadanía. Hoy en día, en el contexto de la pluralidad política y los gobiernos divididos, el problema fundamental consiste en regular a la multiplicidad de actores que, en su búsqueda por el poder, están dispuestos a infringir la normatividad electoral e incluso a romper con los esquemas elementales de una contienda democrática.

En suma, la democracia mexicana todavía enfrenta numerosas limitaciones y dificultades para su consolidación, y ello se refleja inevitablemente en su frágil y deficiente legitimidad ciudadana.

\section{Fuentes de consulta}

Boletín de Prensa del TEPJF, publicado el 24 de agosto de 2012 http:// portal.te.gob.mx/prensa/boletin-prensa/resuelve-tepjf-la-totalidadlos-juicios-inconformidad-el-computo-distrital-la-eleccio, consultada en diciembre 2012.

Instituto Federal Electoral (IFE), Resultados electorales oficiales de 1991-2012, http://www.ife.org. $m x /$ portal/site/ifev2/Estadisticas_y_R esultados_Electorales/consultada en diciembre 2012. 
Esta revista forma parte del acervo de la Biblioteca Jurídica Virtual del Instituto de Investigaciones Jurídicas de la UNAM

Moreno, Alejandro, "Encuesta Reforma: Postelectoral. Deja dudas limpieza de los comicios, pero pide mayoría acatar resultados”, $R e$ forma, 12 julio de 2012.

Sonnleitner, Willibald, Alvarado, Arturo y Sánchez, Arturo (coords.), ¿Inconsistencias, o irregularidades? La calidad de las elecciones de 2012 a la luz de la estadística y geografía de los resultados, así como de la dinámica de las impugnaciones y conflictos (post-)electorales, Informe final del proyecto de observación electoral de El Colegio de México, México, El Colegio de México, 26 de octubre de 2012.

Tribunal Electoral del Estado de México, www.teemx.org.mx, consultada en diciembre 2012.

Tribunal Electoral del Poder Judicial de la Federación (TEPJF), http:// www.trife.gob.mx/, consultada en diciembre 2012.

Woldenberg, José, comunicación personal durante el Seminario sobre las elecciones de 2012, organizado por la Facultad de Ciencias Políticas en la UNAM, el 9 de mayo de 2012. 